\title{
Demokratie und parlamentarische Kontrolle der Entscheidungen im Europäischen Stabilitäts-Mechanismus und Fiskalpakt*
}

\author{
Ulrich Karpen
}

Die geschäftsführende Direktorin des Internationalen Währungsfonds (IWF), Christine Lagarde, soll während einer der unzähligen Verhandlungen zur Errichtung des „Euro-Rettungsschirms“ gesagt haben: „Wenn ich noch einmal das Wort Bundesverfassungsgericht höre, verlasse ich den Raum!" ${ }^{1}$ In der Tat wird in der Rolle des Obersten Gerichtes der Bundesrepublik Deutschland das Spannungsfeld von „Politisierung der Justiz“ und „Juridifizierung der Politik“ überdeutlich. Wochenlang warteten Berlin und die ganze Eurozone, die ganze Europäische Union - wie auf den Spruch des Delphischen Orakels - auf die Karlsruher Entscheidung vom 12. September 2012. ${ }^{2}$ Europa hielt den Atem an, wartete auf ein hochpolitisches Urteil. Ebenso schielten Bundestag und Bundesrat wie Regierung schon seit Beginn der Finanzkrise - wie eigentlich immer, wenn es um die Gesetzgebung geht darauf, „was Karlsruhe wohl sagen wird“, ein Symptom der „Juridifizierung der Politik“. Nun hat es sein Urteil gesprochen, genauer gesagt: in einer einstweiligen Anordnungssache entschieden. Um das Ergebnis vorwegzunehmen: Das Gericht findet sowohl die vorbereitete Ergänzung des supranationalen EU-Rechts, also die Einfügung eines Abs. III in den Art. 136 EU-Vertrag, mit dem Grundgesetz vereinbar, als auch die Intergouvernementalen Verträge zwischen den Euro-Staaten, den ESM (Europäischer Stabilitäts-Mechanismus, „European Financial Stability Mechanism“) und den Fiskalvertrag betreffend. Es verbindet dies allerdings mit der Auflage, bei der Ratifikation zwei völkerrechtlich verbindliche Vorbehalte zu machen: die „Deckelung“ der deutschen Garantiesumme auf 190 Milliarden Euro und die Sicherung parlamentarischer Informations- und Entscheidungsrechte. Die Bundesrepublik müsse erklären, dass sie sich an die Verträge nicht gebunden fühle, sollten sich diese Vorbehalte als unwirksam erweisen.

Bevor im Detail auf die Entscheidung des BVerfG eingegangen wird, muss darauf hingewiesen werden, dass der Europäische Gerichtshof am 27. November 2012 sein Urteil über den ESM-Vertrag gesprochen hat. ${ }^{3}$ Es erging auf Vorlage des Supreme Court der Irischen Republik, wonach Artikel 125 des Vertrages über die Arbeitsweise der Europäischen Union (AEUV), die so genannte „No Bail Out-Klausel“, einer Kreditvergabe durch Mitgliedstaaten an einen anderen Mitgliedstaat nicht entgegenstehe. Voraussetzung sei allerdings, dass der Schuldnerstaat für seine Verbindlichkeiten einstehe und eine tragfähige Haushaltspolitik betreibe. Ferner befasst sich der Richterspruch mit dem Vorwurf, die Europäische Zentralbank (EZB) gebe Finanzhilfen direkt an Mitgliedstaaten, was ihre Kompetenzen übersteige. Der EuGH ist der

* Dieser Beitrag ist die überarbeitete Fassung eines Vortrages, den der Verfasser am 14. September 2012 bei einer europäischen Konferenz im Bundesrat gehalten hat.

1 Zitiert in Melanie Amann, Europa hängt an einer Unterschrift, in: FAS vom 8. Juli 2012, S. 27.

2 BVerfG, 2 BvR 1390/12 vom 12. September 2012, Absatz-Nr. 1 - 215, http://www.bverfg.de/ entscheidungen/rs20120912_2bvr139012.html (Abruf am 11. Januar 2013); das Urteil ist auch abgedruckt in der Neuen Juristischen Wochenschrift 2012, S. 3145 und in der Zeitschrift „Die Öffentliche Verwaltung" (DÖV) 2012, S. 928.

3 Zur Gültigkeit des Euro-Stabilitätsmechanismus (ESM-Vertrag), Urteil des EUGH C 370 vom 27. November 2012, in: Deutsches Verwaltungsblatt 2013, S. $101 \mathrm{ff}$. 
Auffassung, dass die Pflichten, die der Kommission und der EZB im Rahmen des ESM übertragen würden, so wichtig sie auch seien, diese nicht ermächtigten, eigene Entscheidungen zu treffen. Die Verbindlichkeiten, die diese beiden Institutionen eingingen, verpflichteten allein den ESM. Im Endergebnis halten also beide Gerichte den ESM für rechtskonform.

Die Entscheidung des BVerfG vom 12. September 2012 ist das sechste große EuropaUrteil des Gerichtes. Es hat sich vorher schon in fünf Entscheidungen - die wichtigste ist 170 Seiten lang - mit den demokratischen, insbesondere parlamentarischen Möglichkeiten und Grenzen einer Vertiefung der europäischen Einigung, letztlich einer Währungs- und Wirtschaftsunion, beschäftigt: im „Maastricht-Urteil“ (12. Oktober 1993) ${ }^{4}$, „Lissabon-Urteil“ (30. Juni 2009) $)^{5}$, „Griechenland-Urteil“ (7. September 2011) ${ }^{6}$ und im „Minigremium-Urteil“ (28. Februar 2012) ${ }^{7}$, das sich mit Entscheidungsbefugnissen eines Unterausschusses des Haushaltsausschusses des Bundestages in europäischen Finanzierungsangelegenheiten befasste. ${ }^{8}$ Hinzu kommt eine Entscheidung vom 19. Juni 2012, die eine frühzeitige und umfassende Information des Bundestages über Angelegenheiten der EU fordert. ${ }^{9}$ Das Urteil vom 12. September $2012^{10}$ betrifft - wie bemerkt - den ESM und den Fiskalpakt. Über diese Themen soll hier gehandelt werden, zunächst über die Fakten der Europäischen Bankenkrise und die Folgen für die Finanzlage der Staaten der Eurozone, sodann über Fragen der demokratischen Legitimation der in der Rettungsmaschinerie zu treffenden Entscheidungen sowie über grundlegende verfassungsrechtliche und verfassungstheoretische Fragen.

\section{Zur finanziellen Lage des Euro und den gewählten Rettungsinstrumenten}

\subsection{Zahlen, Daten, Fakten}

Die Krise, mit der Europa es zu tun hat, begann 2007 auf dem US-amerikanischen Immobilienmarkt. 2008 brach Lehman-Brothers zusammen. Im selben Jahr beschloss die Gruppe der 20 wichtigsten Industrie- und Schwellenländer (G 20), keine systemrelevante Bank fallen zu lassen. Langsam trockneten die Finanzmärkte aus: der Markt für Staatsanleihen, der Interbankenmarkt, der Aktien- und Devisenmarkt. Es trat sogar eine Liquiditätsverknappung

4 BVerfGE 89, S. 155.

5 BVerfGE 123, S. 267.

6 BVerfGE 129, S. 124.

7 BVerfGE 130, S. 318.

8 Vgl. zu den Urteilen auch Winfried Steffani, Das Demokratie-Dilemma der Europäischen Union. Die Rolle der Parlamente nach dem Urteil des Bundesverfassungsgerichts vom 12. Oktober 1993, in: ders. I Uwe Thaysen, Demokratie in Europa: Zur Rolle der Parlamente, Sonderband zum 25-jährigen Bestehen der ZParl, Opladen 1995, S. 33 - 49, S. 42 ff.; Roland Lhotta / Jörn Ketelhut, Integrationsverantwortung und parlamentarische Demokratie: Das Bundesverfassungsgericht als Agent des „verfassten politischen Primärraums“?, in: ZParl, 40. Jg. (2009), H. 4, S. $864-888$; Dieter Wiefelspütz, Das Primat des Parlaments. Zum Danckert/Schulz-Urteil des Bundesverfassungsgerichts zur Europäischen Finanzstabilisierungsfazilität, in: ZParl, 43. Jg. (2012), H. 2, S. 227 - 250; Birgit Daiber, Das Integrationsverantwortungsgesetz in der Praxis des Deutschen Bundestages, in: ZParl, 43. Jg. (2012), H. 2, S. $293-312$.

9 BVerfG, 2 BvE 4/11 vom 19. Juni 2012, Absatz-Nr. 1 - 172, http://www.bverfg.de/entscheidungen/es20120619_2bve000411.html (Abruf am 11. Januar 2013); Deutsches Verwaltungsblatt 2012, S. 894.

10 Vgl. BVerfG, a.a.O. (Fn. 2). 
ein. 2010 stellten die Euroländer für Griechenland 80 Milliarden Euro bereit, zu zahlen in drei Jahrestranchen. Deutschland ist daran zunächst mit verzinslichen und rückzahlbaren Darlehen von 22 Milliarden Euro beteiligt. Es wurde (substantiell) der Europäische FinanzStabilisierungs-Fonds (EFSF, „European Financial Stability Fund“) und institutionell die Europäische Finanz-Stabilisierungs-Fazilität (EFSF, „European Financial Stability Facility“)11 gegründet. Ihnen folgt der Europäische Finanzstabilisierungs-Mechanismus (ESM, „European Financial Stability Mechanism") und die gleichnamige Institution. ${ }^{12}$

Beide Verträge, EFSF und ESM, sind integrale Bestandteile einer umfassenden Strategie zur Stabilisierung der Lage im Euro-Wirtschaftsgebiet. Diese umfasst außerdem den Vertrag über Stabilität, Koordination und Steuerung der Wirtschafts- und Währungsunion (Fiskalpakt) ${ }^{13}$, die Reform des Stabilitäts- und Wachstumspaktes ${ }^{14}$, die Stärkung der Wettbewerbsfähigkeit durch den Euro-Plus-Pakt ${ }^{15}$ und eine effizientere Finanzaufsicht in der EU. ${ }^{16}$ Insgesamt sind das bedeutende Schritte der dynamischen Entwicklung der EU. Den Anfang bildeten die Europäischen Verträge über den gemeinsamen Markt mit Waren-, Personen-, Dienstleistungs- und Kapitalfreiheit. Hinzu kam 1978 bis 1998 die Europäische Währungseinheit ECU. Ab dem 1. Januar 1999 gilt in der Währungsunion der Euro (Präambel Punkt 8 und Art. 140 AEUV). Es mag gute Gründe dafür geben, diese Währungsunion als Stabilisierungsgemeinschaft zu einer Wirtschafts-, Finanz- und Sozialunion auszubauen, wie es das Bündel der 2012 geschlossenen Verträge tut - mit Schuldenbremse, Bankenaufsicht usw. Fraglich ist allerdings, ob dabei die Haushaltsautonomie der mitgliedstaatlichen Parlamente, ein Kern der nationalen Demokratie, bewahrt werden kann. Der Schritt zur politischen Union mit vollen Rechten des Europäischen Parlaments, Direktwahl des Kommissionspräsidenten, Umwandlung des Rates in eine Länderkammer ist dann nicht mehr weit.

Die finanzielle Belastung ist schon jetzt enorm, wobei keine vollständige Übereinstimmung über die Schulden besteht. ${ }^{17}$ Die kumulierte Haftungssumme für EFSF und ESM beträgt 700 Milliarden Euro, mit einem deutschen Anteil von 190 Milliarden. Die EZB hat im Dezember 2011500 Milliarden Euro Kredite zu einem Zins von einem Prozent ausgegeben, im Februar 2012 weitere 530 Milliarden. Der EZB-Präsident Mario Draghi hat im Juli und August 2012 angekündigt, die Bank werde weiterhin Anleihen, auch solche von finanzschwächeren Ländern aufkaufen, und so hat es der EZB-Rat am 6. September 2012 beschlossen. ${ }^{18}$ Die Staatsschulden der Krisenländer betragen 3,3 Billionen Euro, die Bank-

11 ABl. der EU Nr. L 124/8 und für die Verordnung des Rates ABl. Nr. L 118/1. Dazu BVerfGE 129, S. 124.

12 Deutsches ZustimmungsG, BGBl. 2012 II 983.

13 BGBl. 2012 II 108; dazu Matthias Ruffert, Mehr Europa - eine rechtswissenschaftliche Perspektive, in: Zeitschrift für Gesetzgebung 2013, S. 1, S. 13.

14 ABl. EG Nr. C 236 vom 2. August 1997. Auf S. 1 mit der „3 \% des BIP“-Staatsverschuldungsgrenze.

15 Vom Europäischen Rat am 24./25. März 2011 in Brüssel beschlossen.

16 Vorschlag der Kommission vom 12. September 2012, IP/12/953.

17 So schwirren Zahlen durch den Raum und die Gazetten, vgl. exemplarisch Philip Plickert, EuroVerluste für alle, in: FAZ vom 9. August 2012, S. 9; Konstantinos Simitis, Flucht nach vorn, in: FAZ vom 28. Dezember 2012, S. 9.

18 EZB-Presseerklärung, „Technical features of outright Monetary Transactions“ vom 6. September 2012, http://www.ecb.int/press/pr/date/2012/html/pr120906_1.en.html (Abruf am 11. Januar 2013); vgl. zum Beispiel auch Stefan Ruhkamp / Werner Mussler, Der riskante Kurswechsel der EZB, in: FAZ vom 8. September 2012, S. 12. 
schulden dieser Länder 9,2 Billionen, zum Vergleich: Das deutsche Bruttoinlandsprodukt beträgt 2,5 Billionen, der Bundeshaushalt 2013302 Milliarden Euro.

\subsection{Der Europäische Stabilitätsmechanismus (ESM)}

Das Bundesverfassungsgericht hatte sich in seinem Urteil über den Erlass einer einstweiligen Anordnung zur Verhinderung des ESM vom 12. September $2012^{19}$ mit der möglichen Verfassungswidrigkeit folgender Gesetzentwürfe zu beschäftigen: (1) Gesetz zur Ratifikation des völkerrechtlichen Vertrages zur Änderung des Art. 136 des AEU-Vertrages - Ergänzung um einen Abs. III ${ }^{20}$; (2) Gesetz zum völkerrechtlichen Vertrag zur Einrichtung des ESM ${ }^{21}$ und (3) Gesetz zur finanziellen Beteiligung am ESM ${ }^{22}$. Alle Gesetzentwürfe wurden vom Bundestag und Bundesrat am 29. Juni 2012 verabschiedet, vom Bundespräsidenten am Tag nach der Entscheidung des BVerfG vom 12. September 2012 ausgefertigt und am selben Tage im Bundesgesetzblatt verkündet. Im Zuge der parlamentarischen Beratungen wurden in das ESM-Finanzierungsgesetz auf Vorschlag des Haushaltsausschusses Bestimmungen über eine umfassende Information und Beteiligung von Bundestag und Bundesrat an Europäischen Angelegenheiten eingefügt, die sich in drei Begleitgesetzen niedergeschlagen haben. Darauf wird noch zurückzukommen sein.

Die beschlossene Ergänzung des Art. 136 des AEUV lautet wie folgt: „Die Mitgliedstaaten, deren Währung der Euro ist, können einen Stabilitätsmechanismus errichten, der aktiviert wird, wenn dies unabdingbar ist, um die Stabilität des Euro-Währungsgebietes insgesamt zu wahren. Die Gewährung aller erforderlichen Finanzhilfen im Rahmen des Mechanismus wird strengen Auflagen unterliegen."

Man mag in dieser Bestimmung eine umfassende Ausnahme von der „No Bail Out“Klausel des Art. 125 AEUV sehen, wonach kein Mitgliedstaat für die Schulden eines anderen einzustehen hat, oder eben einen Widerspruch, der Art. 125 leerlaufen lässt. Der EuGH hat, wie erwähnt, die Vereinbarkeit der genannten Bestimmung mit Art. 125 AEUV festgestellt. Stammkapital und Garantiesumme des ESM belaufen sich auf 700 Milliarden Euro, wovon zunächst 80 Milliarden eingezahlt wurden. Die Haftung jedes Mitgliedstaates soll sich unter allen Umständen auf den Anteil am Stammkapital beschränken, für Deutschland also auf 190 Milliarden Euro. Ob nach Art. 8 Abs. V des ESM-Vertrages eine Erhöhung der mitgliedschaftlichen Finanzverpflichtungen möglich ist, ist letztlich unklar. Der scheinbar eindeutige Wortlaut der Regelung mag im Widerspruch zu den ausdrücklich in den Bestimmungen über Kapitalabruf und Verlustausgleich der Art. 9 Abs. II und III, Art. 25 Abs. II ESM-Vertrag geregelten Nachschusspflichten stehen, durch die die Beschränkung des Haftungsrisikos konterkariert würde. Dieser Fall könnte bei Ausfall eines Partners eintreten. Das BVerfG hat diese mögliche Interpretation nicht geteilt, zugleich aber eine völkerrechtliche Klarstellung verlangt. ${ }^{23}$ Für die Vergabe der Mittel ist die internationale Finanzinstitution ESM zuständig,

19 Vgl. BVerfG, a.a.O. (Fn. 2).

20 BGB II 978.

21 Vgl. Deutsches ZustimmungsG, a.a.O. (Fn. 12).

22 BGBl. 2012 II 1918.

23 Vgl. BVerfG, a.a.O. (Fn. 2); dazu auch Andreas Glaser, Die Neuausrichtung der EU-Finanzverfassung durch den Europäischen Stabilitätsmechanismus, in: DÖV 2012, S. 901, S. 904. 
eine Zweckgesellschaft der Euro-Gruppe, also außerhalb der EU stehend. Der ESM gewährt Darlehen und Kreditlinien nach strengen Auflagen, die von einer Troika aus Internationalem Währungsfond, der Europäischen Zentralbank und der Kommission der EU festgelegt werden. Organisatorisch wird der ESM von einem Gouverneursrat (die Finanzminister der Euro-Staaten) und einem Direktorium geleitet (je ein Vertreter jedes Mitgliedslandes). Die Arbeit der ESM-Behörde ist in einem Maße auf Vertraulichkeit angelegt, dass sie der Unabhängigkeit nahe kommt. Nach Art. 32 Abs. V des ESM-Vertrages sind die Archive und sämtliche Unterlagen „unverletzlich“. Artikel 34 bestimmt, dass alle Mitarbeiter, auch nach ihrem Ausscheiden, der beruflichen Schweigepflicht unterliegen. Artikel 35 regelt in großer Ausführlichkeit die persönliche Immunität der Gouverneure und Direktoren des ESM. Fraglich ist zur Zeit, ob dem ESM eine Banklizenz erteilt werden soll. Träte dieser Fall ein, könnte der ESM - wie die EZB - Staatsanleihen kaufen und diese der EZB als Sicherheit für Kredite - also „frisches Geld“ - anbieten. Mit diesen Mitteln könnte der ESM wieder Anleihen kaufen, so dass das Spiel immer weiter gehen könnte. Damit träte die so genannte Hebelwirkung ein, die zweifellos die Inflationsgefahr steigerte. Die EZB kauft jetzt bereits Staatsanleihen auf - rechtlich äußerst umstritten -, wird das weiterhin unbegrenzt tun und möglicherweise auch Eurobonds - also Europäische Staatsanleihen - ausgeben.

\subsection{Der „Fiskalpakt“}

Parallel zur Änderung des Art. 136 AEUV und zum ESM wurde der Vertrag der Eurostaaten über Stabilität, Koordinierung und Steuerung in der Wirtschafts- und Währungsunion geschlossen (SKS-Vertrag, auch „Fiskalvertrag“ genannt; „Treaty on Stability, Coordination and Guidance of Economic and Monetary Union“, „Fiscal Pact“). ${ }^{24}$ Er soll zu einer nachhaltigen Haushaltspolitik und zur Gesundung der Staatsfinanzen in den Euro-Ländern beitragen. Eigentlich als Stärkung der im AEUV angelegten Anforderung an eine stabilitätsorientierte Wirtschafts- und Haushaltspolitik gedacht, wurde er aber - wie der ESM - als völkerrechtlicher Vertrag außerhalb der EU vereinbart, weil sich nicht alle EU-Staaten beteiligen wollten. Beide Verträge stehen aber mit Unionsrecht in unmittelbarem Zusammenhang und können nur mit Blick auf EU-Recht ausgelegt und angewendet werden. Das wichtigste Instrument des Fiskalpaktes ist die Einführung der „Schuldenbremse“ („debt limit“, „debt brakes“), die in vielen Mitgliedsländern für die nationalen Haushalte bereits gilt (vgl. Art. 109 Abs. III, 115 GG) und in Art. 126 Abs. III AEUV in Verbindung mit dem Protokoll Nr. 12 des Vertrages von Amsterdam angelegt ist. Demnach darf das öffentliche Defizit drei Prozent des Bruttoinlandproduktes nicht übersteigen und der gesamte öffentliche Schuldenstand nicht größer als 60 Prozent des Bruttoinlandproduktes sein.

Der „Fiskalpakt“ ist nur ein Teil des Wettbewerbspaktes (SKS-Vertrag). ${ }^{25}$ Dieser enthält weitere Bestimmungen über wirtschaftspolitische Koordinierung und Konvergenz der Wirtschaftspolitiken der Euroländer, über die Steuerung der Eurogebiete („Europakt“). Auch dieser Vertrag ist nicht neu. Er verstärkt nur die Instrumente der früheren Vereinbarungen,

24 Vgl. BGBl. 2012 II 108; Matthias Ruffert, a.a.O. (Fn. 13); dazu jetzt auch Stefan Pilz, Europa auf dem Weg zur Stabilitätsunion? Der Fiskalvertrag im Lichte der Entscheidung des Bundesverfassungsgerichts, in: DÖV 2013, S. 909.

25 Zum Ganzen mit weiteren Nachweisen Matthias Ruffert, a.a.O. (Fn. 13), S. 9 ff. 
wie etwa der „Strategie Europa 2020“, dem „Europäischen Semester“ für eine frühzeitige Überprüfung der nationalen Haushalte usw. Hinzu kommen die im Frühjahr 2012 von Nicolas Sarkozy und Angela Merkel beschlossenen „Leitlinien für eine verstärkte wirtschaftspolitische Koordination der Euro-Staaten“ („Enhanced Economic Policy Coordination in the Euro Area - Main Features and Concepts“) und der „Sixpack“: fünf Verordnungen und eine Richtlinie der EU vom November 2011, betreffend eine Defizitregel zur Korrektur von makroökonomischen Ungleichgewichten. Auch dies ist eine Fortentwicklung des Europäischen Stabilitäts- und Wachstumspaktes. Man kann sagen, dass der Fiskalpakt mitsamt den genannten weiteren Regeln Teil einer integrierten Finanz- und Haushaltspolitik ist; er zieht einen wirtschaftspolitischen Rahmen, kann als Grundbestandteil einer „Eurowirtschaftsregierung“ gelten. Unter dem Dach des „Euro-Paktes“ findet sich die ausdrückliche Verpflichtung der teilnehmenden Staaten, „die im Stabilitäts- und Wachstumspakt der EU enthaltenen Haushaltsvorschriften der EU in nationales Recht umzusetzen “26. Nach Art. 16 des Fiskalvertrages soll der Inhalt innerhalb von fünf Jahren in Gemeinschaftsrecht überführt werden, also für die gesamte EU Geltung erhalten.

\section{Ist der „Rettungsschirm“ mit dem Grundgesetz, insbesondere dem Demokratieprinzip, vereinbar?}

\subsection{Ansatzpunkte verfassungsrechtlicher Kritik}

Dem BVerfG wurde die Frage vorgelegt, ob die geschilderte dynamische Entwicklung der Maßnahmen zur Stabilisierung des Euro mit dem Grundgesetz vereinbar sei. Seit dem 12. September 2012 liegt die Antwort vor, gegeben im einstweiligen Anordnungsverfahren. Die Entscheidung in der Hauptsache wird auf sich warten lassen. Die Klagen gegen das Rettungspaket wurden in zwei Verfahrensarten vorgetragen: 37.000 Bürger hatten Verfassungsbeschwerde eingelegt, weil sie sich durch die Maßnahmen in ihren Grundrechten verletzt sahen, und im Wege einer Organklage hatten MdB Peter Gauweiler (CSU) und die Bundestagsfraktion Die Linke vorgebracht, durch das Handeln des Bundespräsidenten (Unterzeichnung und Verkündung der Gesetze, Ratifikation der Verträge), der Bundesregierung und des Parlaments als Ganzem seien sie in ihren organschaftlichen Rechten verletzt. Es wurde - wie auch in den Maastricht-, Lissabon- und Griechenland-Verfahren - praktisch die ganze Grundstruktur der Verfassung auf die Probe gestellt. Zunächst wurde ein Verstoß gegen das Freiheitsgrundrecht (Art. 2 GG) in Gestalt der politischen Freiheit zu demokratischer Teilnahme an staatlichen Entscheidungen gerügt, auch verkörpert im grundrechtsgleichen Individualrecht aus dem Demokratieprinzip des Art. 38 GG. Sodann wurde die Eigentumsgarantie (Art. 14 GG) - Geldwertschwund - in Stellung gebracht. Auf die angebliche Verletzung des Widerstandsrechtes des Bürgers (Art. 20 Abs. IV GG), das greift, wenn alle rechtsstaatlichen Hilfen versagen, braucht nicht eingegangen zu werden, weil offensichtlich ist, dass der Weg zum BVerfG eben noch offen stand, staatliche Hilfe also zu erwarten war und ist. Der Vorwurf, die Euro-Rettungspolitik habe bisher keine Grundlage im EU-Recht und verstoße gegen die „No Bail Out“-Klausel (Art. 125 AEU), bedeute im Übrigen - so

26 Beschluss des Europäischen Rates vom 24./25. März 2011, EUCO 10/1/11 REV1, S. 19. 
die Beschwerdeführer - eine fundamentale Neuausrichtung der Währungsunion und müsse vom EuGH geprüft werden. Wie erwähnt, hat der EuGH sich in seiner Entscheidung vom 27. November 2012 mit diesen Fragen beschäftigt und die Anträge zurückgewiesen. ${ }^{27}$ Das Bundesverfassungsgericht hat die Klagen nur in Bezug auf das Demokratieprinzip, also Art. 38, 20 Abs. I und 19 Abs. III GG, für zulässig und teilweise begründet erklärt.

Kennzeichnend für das Verfahren vor dem BVerfG und vielleicht für die deutsche Verfassungsrechtsprechung überhaupt - im Vergleich zu höchsten Gerichten anderer Länder - ist, dass die Strukturbestimmungen der staatlichen Grundordnung ins Feld geführt werden, um die Verträge zu Fall zu bringen. Das gilt nicht nur für die Demokratie, sondern auch für die anderen „Großbegriffe“ wie Rechtsstaat und Sozialstaat. Sogar die Verfassungsidentität des Grundgesetzes und damit die deutsche Staatlichkeit werden von den Beschwerdeführern in Gefahr gesehen. ${ }^{28}$ Die Euro-Verträge erdrückten als Exekutivgesetzgebung das Parlament und gefährdeten die rechtsstaatliche Gewaltenteilung auf nationaler Ebene. Auf europäischer Ebene, wohin Finanzkompetenzen verschoben würden, sei die Gewaltenteilung ohnehin unzureichend organisiert. Auch sei in der EU die grundrechtlich rechtsstaatliche Kontrolle nicht voll ausgebildet. Der Sozialstaat in Deutschland sei durch die Europäische Wettbewerbsordnung - vor allem im „Euro-Pakt“ - und den Übergang von einer Währungsunion in eine Haftungs- und Transfergemeinschaft gefährdet. Vor allem aber sei die demokratische Grundstruktur nachhaltig betroffen oder schon verletzt, vor allem die parlamentarische Demokratie, im Besonderen in Gestalt der Haushaltsautonomie und Budgetkontrolle durch das Parlament. Diese gehörten zum Kern der souveränen Staatlichkeit überhaupt, und folglich stehe die Identität der Bundesrepublik auf dem Spiel.

\subsection{Verstoß gegen das Demokratieprinzip?}

Auf die Entfaltung des Demokratiegebotes, die Beteiligung des demokratisch gewählten Parlaments an der Ausübung der Staatsgewalt und vor allem die parlamentarische Kontrolle allen Staatshandelns verwendet das BVerfG in allen sechs genannten Urteilen das vielfältige Arsenal seiner Auslegungsmethoden. Das Urteil vom 12. September 2012 geht hier besonders ins Detail.

Demokratie (Art. 20, 38 GG) ist nicht nur ein konstituierendes Staatsprinzip, sondern ein grundrechtsgleiches Recht des Bürgers - nicht nur im formellen Sinne als aktives und passives Wahlrecht, sondern zugleich als materielles Recht, das dem Bürger als Mitträger der Staatsgewalt den Anspruch auf freie und gleiche Teilhabe an der demokratisch ausgeübten Staatsgewalt verbürgt. ${ }^{29}$ Er hat als Einzelner ein klagbares Recht auf Einhaltung des Demokratiegebotes, einschließlich der verfassungsgebenden Gewalt des Volkes. Diese Facetten sind nach Art. 79 Abs. III GG unveränderlich und unantastbar. Das demokratische Grundrecht des Bürgers ist also nicht nur ein klassisches Abwehrrecht („status negativus“) gegen Demo-

27 Vgl. Urteil des EUGH, a.a.O. (Fn. 3).

28 Verfassungsbeschwerden des MdB Peter Gawweiler vom 29. Juni 2012, Umdruck S. 35 ff., und von Albrecht Schachtschneider vom selben Tage, Umdruck S. 54 ff.; Zusammenfassung der Argumentation der Beschwerdeführer im Urteil des BVerfG vom 12. September 2012, vgl. BVerfG, a.a.O. (Fn. 2); DÖV, S. $934 \mathrm{ff}$.

29 BVerfGE 99, S. 1, S. 10; BVerfGE 108, S. 251, S. 266; BVerfGE 112, S. 118, S. 133. 
kratieentzug, sondern auch ein (positives) Teilhaberecht an der Ausübung der Volksherrschaft im Staat. Vermittelt nimmt der Bürger also etwa an der Verabschiedung von Gesetzen und Haushalten sowie der Kontrolle ihrer Durchführung durch das Parlament teil. Gehen diese Kompetenzen verloren, ist nichts mehr mitzuentscheiden und zu kontrollieren. Gibt der Staat Kompetenzen „nach oben“, nach Europa, ab, muss der Bürger auch an dieser Ausübung durch suprastaatliche und intergouvernementale Organe beteiligt werden. Da er sein demokratisches Grundrecht - außer durch Formen der direkten Demokratie, wie Abstimmungen oder Volks- und Bürgerentscheide (Art. 20 Abs. II GG) - wesentlich durch die Wahl des Parlaments wahrnimmt, muss dieses auch über die Mitgliedschaft in supranationalen Organisationen, über ihren Fortbestand und ihre Entwicklung bestimmen.

Nun wird gegen den ESM eingewandt, der Deutsche Bundestag habe durch Zustimmung seine Haushaltsautonomie und -kontrolle weitgehend aufgegeben. ${ }^{30}$ Deutschland beteiligt sich am ESM-Rettungsschirm mit 190 Milliarden Euro. Bei einem Jahreshaushalt (2013) von 302 Milliarden verlöre, so die Kläger, das Land seine Budgethoheit bis zu 60 Prozent. Durch den ESM sei die Union - der Euroraum - keine sektorale Wirtschaftsgemeinschaft mehr, sondern eine Wirtschafts-, Finanz- und Sozialunion. In einer solchen Union sei die Haushaltsautonomie nur dann gewährleistet, wenn Finanzhilfen unter Mitgliedstaaten verboten seien, wie es die „No Bail Out“-Klausel bestimmt. Die Bundesrepublik hätte ihre Währungshoheit nicht auf die EU und den ESM übertragen dürfen, ohne sicherzustellen, dass das Haushaltsrecht in Deutschland bleibt. Gegen diese Sicht der Dinge wendet das Verfassungsgericht ein ${ }^{31}$, dass die EU - auch in Währungsangelegenheiten - eben keine Kompetenz-Kompetenz habe (Art. 5 EUV). Sie besitze nur Einzelermächtigungen, und dem ESM seien nur begrenzte Mittel übertragen. Im Übrigen seien beide Institutionen der Achtung der verfassungsrechtlichen Identität der Mitgliedstaaten, der loyalen Zusammenarbeit, der Subsidiarität und der „ultra-vires“-Kontrolle verpflichtet. Das Gegenargument lautet: Es gebe eine "Vertragsabrundungskompetenz" der EU, eben die Flexibilitätsklausel (Art. 352 AEUV) ${ }^{32}$, eine Blankettermächtigung (Art. $352 \mathrm{AEUV)} \mathrm{und} \mathrm{das} \mathrm{so} \mathrm{genannte} \mathrm{Brückenver-}$ fahren (Art. 48 VI EUV), wonach der Rat nicht mehr einstimmig, sondern mit qualifizierter Mehrheit über Vertragsänderungen entscheidet. Allerdings können die beiden letztgenannten Kompetenzklauseln nur mit Zustimmung der mitgliedstaatlichen Parlamente, also auch des Bundestages, wahrgenommen werden.

Mit diesen Argumenten hat sich das BVerfG ausführlich auseinandergesetzt. ${ }^{33}$ Es vertritt im Wesentlichen die Auffassung, die ESM-Regelungen seien demokratiekonform. Es sei richtig, dass der Schuldenstand einen erheblichen Umfang angenommen habe. Der Bundestag bleibe aber der Ort, an dem eigenverantwortlich über Einnahmen und Ausgaben entschieden werde, auch im Hinblick auf internationale und europäische Verbindlichkeiten. Die Schuldenunion, die sich entwickele, löse sich allerdings von dem bisherigen Prinzip der Eigenständigkeit der nationalen Haushalte. Es müsse deshalb im Blick auf die Rechte des Parlaments dafür Sorge getragen werden, dass keine Automatik der Schuldenerhöhung eintrete, keine dynamische Entwicklung außerhalb von Entscheidung und Kontrolle des Parlaments. Das sei aber gewährleistet und müsse - so die erste in der Entscheidung ausgespro-

30 Vgl. Peter Gauweiler, a.a.O. (Fn. 28), S. 35 ff.; Albrecht Schachtschneider, a.a.O. (Fn. 28), S. 68 ff.

31 Vgl. BVerfG, a.a.O. (Fn. 2), S. 27.

32 Oder „Kompetenzergänzungsklausel“.

33 Vgl. BVerfG, a.a.O. (Fn. 2), S. 27, S. 31. 
chene Auflage des BVerfG für die Ratifikation der Verträge - zusätzlich gesichert werden. Zunächst enthalte Art. 136 III AEUV, der an sich eine Ausnahme von der Stabilitätsunion sei, nur die Möglichkeit, Mechanismen zur Eurorettung völkerrechtlich einzurichten. An der Umsetzung durch völkerrechtliche Verträge sei der Bundestag beteiligt, also seien seine Rechte gewahrt. Das Gericht tut aber ein Übriges, um einer Verselbständigung der Schuldenunion vorzubeugen. Es verpflichtet die Bundesregierung, bei der Ratifikation des Vertrages den verbindlichen Vorbehalt zu machen, dass die Haftung der Bundesrepublik strikt bei 190 Milliarden Euro bleiben müsse. ${ }^{34}$ Das steht zwar schon ausdrücklich in Art. 8 Abs. V ESMV, aber Art. 9 II über den revidierten Kapitalabruf, der nicht ganz eindeutig ist, veranlassten die Verfassungsrichter zu dieser klärenden Vorsichtsmaßnahme.

Die Linie mangelnder demokratischer Teilnahme an Budgetentscheidungen wird von Klägern und Gericht noch fortgeführt und vertieft. ${ }^{35}$ Es wird argumentiert, dass durch die zwischenzeitliche dynamische Entwicklung der Union und die Errichtung des ESM so weitgehende Kompetenzen abgegeben worden seien, dass das notwendige demokratische Legitimationsniveau der Völker der Mitgliedstaaten unterschritten sei. Zentrale politische Entscheidungen würden nicht mehr von den Staaten getroffen. Diese seien aber „Herren der Verträge“, und das müsse so bleiben, solle "demokratische Volksherrschaft", demokratische Legitimität bewahrt werden, wie es nach dem Grundgesetz (Art. 23 Abs. I) erforderlich sei. Die Organe der EU und auch des ESM müssten aus der mitgliedstaatlichen Demokratie legitimiert sein, für die Union allenfalls „doppelt legitimiert“ infolge der Mitwirkung des Europäischen Parlaments. Durch Kompetenzüberschreitungen - etwa Mehrheitsentscheidungen des Rates oder der ESM-Organe - oder einzelne „ausbrechende Rechtsakte“ werde das demokratische Legitimationsband zu den nationalen Völkern und Parlamenten zerschnitten. Die mangelnde legitimierende Rückbindung an die nationalen Parlamente werde auch nicht durch die Beteiligung des Europäischen Parlaments wettgemacht. Es sei nicht nach dem demokratischen Grundsatz gleicher, also erfolgswertgleicher Wahl gewählt. Es finde kein Parteienwettbewerb statt, und das Prinzip wechselnder Mehrheiten funktioniere nicht. Auch der ESM sei nicht ausreichend legitimiert. Gouverneursrat und Direktorium hätten Pflichten, Vorrechte und Befreiungen, seien sogar immun. Unterlagen, die sie anlegten und fortführten, seien „unverletzlich“, also geheim und damit der Kontrolle durch nationale Parlamente nicht zugänglich. Im Dienst und auch nach ihrem Ausscheiden seien Gouverneure und Direktoren der beruflichen Schweigepflicht unterworfen. Das entziehe sie der parlamentarischen Verantwortung und sei ein Verstoß gegen das Demokratiegebot der Art. 20 und Art. 23 Abs. I GG. Darauf ist das BVerfG mit seiner zweiten Auflage für das Ratifikationsverfahren eingegangen. Mit der Ratifikation sei der Vorbehalt zu verknüpfen, dass diese Vorrechte nicht so verstanden werden dürften, dass sie dem Kontrollrecht des Parlaments entgegenstünden. ${ }^{36}$

Was die Bedenken der Antragsteller hinsichtlich der unzureichenden demokratischen Binnenorganisation der EU angeht, bot der Fall keinen Anlass für das Gericht, in eine Erörterung einzutreten. Das ändert aber nichts daran, dass die Frage nach Zusammensetzung und Kompetenzen des Europäischen Parlaments eine Kernfrage der supranationalen Verfasstheit Europas ist. Der Vorwurf, das Parlament sei nicht demokratisch gewählt, wird seit 
langem und jetzt verschärft erhoben. ${ }^{37}$ Dem ist allerdings entgegenzuhalten, dass die deutsche innerstaatliche parlamentarische Demokratie nicht als Maßstab „der Demokratie“ schlechthin dienen kann. Schon Präsidialsysteme und Staaten mit Mehrheitswahlrecht haben andere Vorstellungen von Legitimität. Die Abweichungen der unionsdemokratischen Legitimität sind auf die Besonderheiten des Staatenverbundes zurückzuführen. Art. 23 Abs. I Satz 1 GG verlange hinsichtlich der Einhaltung demokratischer Grundsätze durch die Europäische Union keine „strukturelle Kongruenz" mit der Ordnung des deutschen Verfassungsrechtes; geboten sei ,jedoch eine dem Status und der Funktion der Union angemessene demokratische Ausgestaltung "38. Die ist aber gegeben. Dass Abweichungen vom Prinzip "one person - one vote“ erforderlich sind, zeigt folgendes Beispiel: Luxemburg hat mit 500.000 Einwohnern im Europäischen Parlament sechs Abgeordnete. Bliebe man bei dieser Relation, müsste das Europäische Parlament bei einer halben Milliarde Europabürgern 6.000 Abgeordnete umfassen. Man muss also gegenwärtig tolerieren, dass Angehörige eines kleinen Staates das zwölffache Stimmgewicht der Bürger eines großen haben. Nicht zuletzt wegen dieses Befundes wird es ab 2017 im Rat doppelte Mehrheiten geben.

\subsection{Verlust von Kernkompetenzen und Staatlichkeit?}

Noch einen Schritt weiter gehen die Antragsteller, die in der Entwicklung von ESM und EU von der Währungsunion zur "Schuldenunion“ nicht nur den Verlust von Kernkompetenzen und legitimierter Demokratie sehen, sondern sogar die Aufgabe deutscher Staatlichkeit. ${ }^{39} \mathrm{Ge}-$ meint ist eigentlich der Verlust souveräner Staatlichkeit zugunsten der Mitgliedschaft in einem europäischen Bundesstaat. Artikel 20 Abs. II Satz 1 GG mit der Formulierung „,alle Staatsgewalt geht vom Volke aus" sei an sein Ende gekommen. Nun kann man dem mit gutem Grund entgegenhalten, dass die Bundesrepublik nach Art. 50 EUV ausdrücklich ein Recht des Austritts aus der EU hat. Auch im System des Rettungsschirms ist der "point of no return“ noch nicht erreicht. Zwar kann Deutschland nicht aus dem ESM austreten. Nach Art. 4 Abs. II des ESM-Vertrages kann aber bei Entscheidungen, die die haushaltspolitische Gesamtverantwortung betreffen, der deutsche Vertreter im Gouverneursrat ohne entsprechenden Beschluss des Bundestages nicht zustimmen. Erhält er die Zustimmung des Parlaments nicht, muss er sein Veto einlegen, das wegen des deutschen Stimmenanteils von 27 Prozent verhindernde Wirkung hat. Damit wird letztlich die mitgliedstaatliche Souveränität gewahrt. Diese Auffassung teilt auch das BVerfG, und zwar nicht nur in seiner Entscheidung vom 12. September 2012.40

Noch wichtiger ist allerdings, dass von „Entstaatlichung“ nur reden kann, wer die ausdrückliche und wiederholte Entscheidung des Grundgesetzes für eine „offene Staatlichkeit“

37 Zusammenfassend Peter Graf Kielmansegg, Wenn die Verfassung schweigt, in: FAZ vom 20. September 2012, S. 8.

38 Vgl. BVerfGE 123, S. 267, S. 365 („Lissabon-Urteil“); Volker Röben nennt das „strukturelle Kompatibilität“, ders., Außenverfassungsrecht. Eine Untersuchung zur auswärtigen Gewalt des offenen Staates, Tübingen 2007, S. 321.

39 Vgl. Albrecht Schachtschneider, a.a.O. (Fn. 28), S. 73; dazu (kritisch) Matthias Herdegen, in: Theodor Maunz / Günter Dürig (Hrsg.), Grundgesetz. Kommentar, Loseblattsammlung, München, Stand: November 2012, Art. 146, Rn. 57.

40 Vgl. BVerfG, a.a.O. (Fn. 2), Abs. 131; vor allem BVerfGE 123, S. 267, S. 371 („Lissabon-Urteil“); BVerfGE 130, S. 318, S. 345 („Minigremium-Urteil“). 
übersieht. ${ }^{41}$ In der Präambel ist von dem Willen die Rede, das Land solle „als gleichberechtigtes Glied in einem vereinten Europa dem Frieden in der Welt ... dienen“. Artikel 23 verpflichtet die Bundesrepublik zur „Verwirklichung eines vereinten Europas“ mit demokratischen, rechtsstaatlichen, sozialen und föderativen Grundsätzen sowie dem Schutz von Grundrechten. Artikel 24 (Übertragung von Hoheitsrechten auf Einrichtungen eines kollektiven Sicherheitssystems) wie Art. 25 GG (Anerkennung der allgemeinen Regeln des Völkerrechts als Bestandteile des Bundesrechts) bekennen sich zur Supra- und Internationalität. Dabei bleibt Deutschland ein Staat mit Staatsvolk, Staatsgebiet und Staatsgewalt, eine handlungsfähige politische Gemeinschaft. Es ist keineswegs ausgemacht, dass der unantastbare Verfassungskern des Art. 79 Abs. III GG verletzt ist: Bundesstaatlichkeit, Menschenwürde und Menschenrechte sowie demokratische Rechtsstaatlichkeit. Bei der geplanten Änderung des Europarechts, dem ESM und dem Fiskalpakt handelt es sich eher um die Fortentwicklung des Stabilitäts- und Wachstumspaktes. Schon gar nicht ist eine „stillschweigende Ablösung “ des Grundgesetzes zu konstatieren, die den „pouvoir constituant“, das Volk als die Quelle einer neuen Verfassung, auf den Plan riefe (Art. 146 GG). Von einem Verlust der Identität der deutschen Verfassung kann schwerlich die Rede sein. ${ }^{42}$

\section{Werden die parlamentarischen Kompetenzverluste durch Partizipation an der europäischen Entwicklung ausgeglichen?}

Die durch die europäische Dynamik bereits eingetretenen und weiterhin drohenden Kompetenz-, Demokratie- und Legitimitätseinbußen von Bundestag und Bundesrat können durch Transparenz, Beteiligungsrechte und die verfahrensmäßige Stärkung der Subsidiarität wettgemacht werden. Verfassungsrechtliche Quellen sind der „Europaartikel“ 23 GG und $\$ 4$ Abs. I des ESM- Finanzierungsgesetzes. Einzelheiten sind in drei Begleitgesetzen ${ }^{43}$ geregelt, die zum Teil noch in der parlamentarischen Beratung des ESM-Gesetzes geändert worden sind: das Gesetz über die Zusammenarbeit von Bundesregierung und Deutschem Bundestag in Angelegenheiten der Europäischen Union (EUZBB), das Gesetz über die Zusammenarbeit von Bund und Ländern in Angelegenheiten der Europäischen Union

41 Dazu insbesondere Christoph Möllers, Gewaltengliederung, Legitimation und Dogmatik im nationalen und internationalen Rechtsvergleich, Tübingen 2005, S. 253 ff.; ders., Demokratie - Zumutungen und Versprechen, Berlin 2008, S. 82 ff. („Demokratie jenseits des Staates“).

42 Allenfalls ließe sich sagen, dass es in der Fortentwicklung der deutschen Demokratie an der Zeit wäre, die unmittelbare Demokratie in Gestalt von Volksentscheiden zu stärken. Vgl. Mario Marini, Wenn das Volk (mit-)entscheidet, Wechselbeziehungen und Konfliktlinien zwischen direkter und indirekter Demokratie als Herausforderung für die Rechtsordnung, Berlin 2011, S. 96. $\mathrm{Zu}$ denken wäre an eine Ergänzung des Europaartikels 23 GG um eine Formulierung: „Zustimmungsgesetze zu Verträgen oder Vertragsänderungen in Angelegenheiten der Europäischen Wirtschafts- und Währungsunion, bedürfen der Bestätigung durch Volksentscheid. Das Nähere regelt ein Bundesgesetz", so der Vorschlag von Hans Peter Schneider, Das Volk überzeugen, in: FAZ vom 9. August 2012, S. 6. Das wäre eine Angleichung deutschen Rechts an die Verfassungslage in anderen Mitgliedsländern der EU und würde das Volk instand setzen, einer weiteren Vertiefung der europäischen Einigung entgegenzutreten, wenn es das denn wollte - so, wie 2005 Frankreich und die Niederlande eine Europäische Verfassung ablehnten.

43 BGBl. I, S. 3022 vom 22. September 2009; jetzt in der Fassung BGBl. I, S. 3822 vom 22. September 2012. 
(EUZBLG) und das Gesetz über die Integrationsverantwortung des Bundestages und des Bundesrates in Angelegenheiten der Europäischen Union (IntegrationsverantwortungsG), die schon 1993 beziehungsweise 2009 erlassen worden sind.

Artikel 23 Abs. II GG lautet: „In Angelegenheiten der Europäischen Union wirken der Bundestag und durch den Bundesrat die Länder mit. Die Bundesregierung hat den Bundestag und den Bundesrat umfassend und zum frühestmöglichen Zeitpunkt zu unterrichten." Absatz III formuliert: „Die Bundesregierung gibt dem Bundestag Gelegenheit zur Stellungnahme vor ihrer Mitwirkung an Rechtsakten der Europäischen Union. Die Bundesregierung berücksichtigt die Stellungnahmen des Bundestages bei den Verhandlungen." 2008 wurde Art. 23 Abs. Ia ins Grundgesetz eingefügt: „Der Bundestag und der Bundesrat haben das Recht, wegen Verstoßes eines Gesetzgebungsaktes der EU gegen das Subsidiaritätsprinzip vor dem Gerichtshof der EU Klage zu erheben." Ferner regelt $\$ 4$ des ESM-FinanzierungsG $G^{44}$ den deutschen Parlamentsvorbehalt für Entscheidungen im ESM: „In Angelegenheiten des ESM, die die haushaltspolitische Gesamtverantwortung des Deutschen Bundestages betreffen, wird diese vom Plenum des Bundestages wahrgenommen. Die haushaltspolitische Gesamtverantwortung ist insbesondere betroffen (...)." Damit sind die drei Instrumente benannt, mit denen die entgangenen demokratisch legitimierten Entscheidungen ausgeglichen werden sollen: Information, Zuständigkeitsausübung durch Stellungnahmen etc. und Subsidiaritätsklage.

(1) Nach $\$ \$ 4 \mathrm{ff}$. EUZBB unterrichtet die Regierung den Bundestag in sachlicher, zeitlicher und förmlicher Hinsicht umfassend und zum frühestmöglichen Zeitpunkt. Es versteht sich von selbst, dass das Parlament diesen Informationsanspruch als Träger der Budgethoheit in erster Linie gegenüber den ESM-Organen hat. Das hat das BVerfG bestätigt und das Unterrichtungsrecht auf Art. 38 und 79 Abs. III GG gestützt. Die Information des Bundestages durch die Regierung geschieht mündlich - im Plenum und Ausschüssen - oder/und durch Dokumente. Erläutert werden Regelungsinhalt, Alternativen und Kosten. Die Unterrichtung enthält auch eine Rechtsfolgenabschätzung. Die gleiche Informationsverpflichtung hat die Bundesregierung nach $\$ 2$ EUZBLG gegenüber dem Bundesrat.

(2) Die Zuständigkeitsausübung von Bundestag und Bundesrat nach Art. 2 des deutschen Zustimmungsgesetzes zum ESM enthält Zustimmungsvorbehalte des deutschen Parlaments für wichtige Entscheidungen, etwa zur Erhöhung des Stammkapitals für Mitentscheidungsrechte des deutschen Gouverneurs und Direktors. Diese werden in $\$ \$ 4 \mathrm{ff}$. des ESM-Finanzierungsgesetzes aufgezählt. Diese Rechte fügen sich durchaus in die Parlamentsvorbehalte im Unionsrecht ein: im Brückenverfahren vereinfachter Vertragsänderung (Art. 48 VII EUV), bei der Auslegung von Blankettvorschriften, im „Vertragsabrundungsverfahren“ (Art. 308, 352 AEUV). Sie sind im deutschen Integrationsverantwortungsgesetz (2009/2012) spezifiziert worden. Beide Häuser des Parlaments haben nach Art. 23 Abs. III GG, $\$ 9$ EUZBB und $\$ 3$ EUZBLG das Recht zu Stellungnahmen. Diese muss die Bundesregierung, was den Bundesrat angeht, „einfach“ oder „maßgeblich“ beachten, je nach Art der betroffenen Gesetzgebungskompetenzen. Nach $₫ 5$ des ESM-Finanzierungsgesetzes wird der Haushaltsausschuss des Bundestages regelmäßig beteiligt. Die Bundesregierung muss die Stellungnahme berücksichtigen. Im „Griechenland-Urteil“ vom September $2011^{45}$ hat das BVerfG diese Bestimmung so interpretiert, dass die Berücksichtigungspflicht („Bemühen um Ein- 
vernehmen") die Bundesregierung verpflichtet, die vorherige Zustimmung des Ausschusses einzuholen. Nach $\$ 6$ EUZBLG sind auf Verlangen Vertreter der Länder zu den Brüsseler Beratungen hinzuzuziehen, wenn Länderangelegenheiten auf der Tagesordnung stehen. In diesen Fällen können nach $\$ 4$ im Übrigen auch Ländervertreter mit der Bundesregierung zusammenarbeiten, wenn es um die Festlegung der deutschen Verhandlungsposition geht.

(3) Bundestag und Bundesrat erfahren eine verfahrensmäßige Verstärkung ihrer Position, das europäische Verfassungsprinzip der Subsidiarität (Art. 5 EUV) zur Geltung zu bringen (Art. 23 Abs. I a GG). Beide Organe können Subsidiaritätsklage beim EuGH erheben. Im Bundestag ist dies als Minderheitsrecht ausgestaltet. Klageberechtigt ist ein Viertel der Abgeordneten.

\section{Vor der Hauptsacheentscheidung des Bundesverfassungsgerichtes}

\subsection{Die Anhörung am 16./17. Juni 2013}

Vor Eintritt in einige abschließende Beobachtungen ist festzuhalten, dass das BVerfG gegen den Fiskalpakt keinerlei Einwände erhoben hat. Die Regelungen, gerade zur „Schuldenbremse“, entsprächen in hohem Maße den Stabilitätsvorschriften des Grundgesetzes (Art. 109, 109a, 115).

Das Urteil des BVerfG vom 12. September 2012 ist in einem einstweiligen Anordnungsverfahren ergangen. Die Beschwerdeführer wollten durch die beantragte Verfügung das Inkraftsetzen des ESM verhindern. Das Gericht hat an einigen Stellen ${ }^{46}$ ausgeführt, dass es in einem kursorischen Verfahren mit nur summarischer Prüfung der Rechtsfragen nicht alle Probleme mit gebotener Gründlichkeit untersuchen könne und ausdrücklich auf die Entscheidung im Hauptsacheverfahren verwiesen. Zur Vorbereitung dieser Entscheidung hat es am 16./17. Juni 2013 eine zweitägige mündliche Anhörung abgehalten. Dabei ging es vorwiegend um die Frage, ob die Europäische Zentralbank Staatsanleihen der Euro-Krisenstaaten auf dem Sekundärmarkt erwerben dürfe, wie es deren Präsident Mario Draghi am 6. September 2012 verkündet hatte. Immerhin stellte das Gericht vorbehaltlich der endgültigen Entscheidung im Anordnungsverfahren fest: „[E]in Erwerb von Staatsanleihen am Sekundärmarkt durch die Europäische Zentralbank, der auf von den Kapitalmärkten unabhängige Finanzierung der Haushalte der Mitgliedstaaten zielte, ist als Umgehung des Verbotes monetärer Haushaltsfinanzierung ebenfalls untersagt. “ 47

\subsection{Zur Funktion des BVerfG im deutschen und europäischen Regierungssystem}

Das angespannte Warten aller deutschen Staatsorgane auf das Urteil des Bundesverfassungsgerichts vom 12. September 2012 und die von ihm hervorgerufenen hektischen Reaktionen haben - wieder einmal - eine breite Diskussion seiner Rolle im Regierungssystem Deutschlands eröffnet. Selten hat sich deutlicher gezeigt, wie weit die Karlsruher Richter ausgreifen, um Zugriff auf auch hochpolitische Materien zu nehmen. Das gilt vor allem für die Anwen-

46 Vgl. BVerfG, a.a.O. (Fn. 2), S. 41, S. 42.

47 Vgl. ebenda, S. 39. 
dung von Auslegungsmethoden, die gelegentlich auf das höchste Seil der Theorie führen. Gewiss steht dem BVerfG die „political question doctrine“ nicht zur Verfügung. Es muss politische Fragen aus der Verfassung beantworten. Ein spöttischer Staatsrechtslehrer hat einmal gesagt, für das Gericht - und nicht nur für das Gericht - sei das Grundgesetz ein „juristisches Weltenei“, aus dem jede notwendige Entscheidung als Rechtssatz herauskomme. ${ }^{48}$ Das BVerfG muss die Verfassung auslegen - „we are under the constitution, but the constitution is what the judges say it is" (so der US-amerikanische Chief Justice Charles E. Hughes $^{49}$ ) - aber es tut dies - wie in Europasachen - in einzigartiger Weise. Das gilt besonders für den Demokratiebegriff, der überdeutlich im Blick auf deutsches Verfassungsrechtsverständnis und deutsche Verfassungstheorie interpretiert wird. Es gibt aber nicht nur ein richtiges Modell der Demokratie. Vergleichbares gilt für den Staatsbegriff. Das BVerfG lädt vor allem im Lissabon-Urteil von $2009^{50}$ - das Gebot demokratischer Staatlichkeit mit einer Verfassungsidentität auf, die aus begrifflichen, komparativen, genetischen und historischen Gründen kaum mit dem Grundgesetz vereinbar ist. Ferner halten sich die Richter in ihrer Europarechtsprechung gerade bei der Folgenabwägung der getroffenen oder zu treffenden Euro-Stabilisierungsmaßnahmen nicht immer an die von ihnen selbst im Grunde anerkannten Schranken. Im „politischen Kernbereich“ gibt es einen großen Einschätzungsspielraum von Regierung und Parlament. Für das BVerfG kann es nur darum gehen, die evidente Überschreitung äußerster Grenzen zu kennzeichnen. Das Resultat des teils notwendigen, teils gewollten weiten Ausgreifens des Gerichts in das politische Feld ist eine „Politisierung der Justiz“ („gouvernement des juges“) und eine „Juridifizierung der Politik“, indem das Parlament auf „Karlsruhe schielt“ und überstimmte Parlamentsminderheiten sogleich „nach Karlsruhe“ gehen. Dennoch sind nach einer neuen Umfrage 68 Prozent der Bürger froh, dass die verfassungsrechtliche Zulässigkeit des ESM vom BVerfG überprüft wurde. ${ }^{51}$ Nur 17 Prozent erfüllt ein derartig starker Einfluss des Gerichts auf politische Entscheidungen mit Unbehagen. Justizgläubigkeit? Kompliment für Karlsruhe? Misstrauensvotum für Berlin?

Die wichtigste Frage ist natürlich nicht die deutsche Verfasstheit und Seelenlage, sondern die Frage an alle Europäer: Welches ist die Finalität Europas? ${ }^{52}$ Wohin soll die Reise gehen? Europa startete mit der asymmetrischen Konstruktion einer vollständigen Vergemeinschaftung der Währungspolitik bei gleichzeitiger Zuständigkeit der Mitgliedstaaten für die Wirtschaftspolitik und ist nun auf dem Weg in eine Wirtschaftsunion. Wollen wir eine Politikunion? Die deutsche Verfassung erlaubt jedenfalls eine „offene Staatlichkeit“ und auch die „Politische Union“, wenn die Bürger sie denn wollen - und Karlsruhe sie zulässt.

48 Vgl. Ernst Forsthoff, Der Staat der Industriegesellschaft, München 1971, S. 144.

49 Charles E. Hughes, Addresses and Papers of Charles Evan Hughes, New York 1908, S. 193.

50 BVerfGE 123, S. 267.

51 Vgl. Renate Köcher, Das Bollwerk, in: FAZ vom 22. August 2012, S. 10.

52 Vgl. Barbara Lippert, Die EU zusammenhalten - aber wie?, Arbeitspapier der Stiftung Wissenschaft und Politik, Berlin 2013. 